\title{
Aortic stenosis and CT calcium scoring: is it for everyone?
}

\author{
Timothy R G Cartlidge, Tania A Pawade, Marc R Dweck
}

The evaluation of aortic stenosis (AS) severity is routinely performed using echocardiography. Current guidelines recommend integrating data with respect to the peak aortic valve jet velocity, the mean transvalvular gradient and the aortic valve area (AVA) calculated by the continuity equation. ${ }^{1}$ For the majority of patients this assessment works well. However in a third of subjects there is discrepancy in the results provided by these different measures. ${ }^{2}$ Most commonly this takes the form of an AVA $<1.0 \mathrm{~cm}^{2}$ indicating severe stenosis but a peak velocity $<4 \mathrm{~m} / \mathrm{s}$ and mean gradient $(\mathrm{MG})<40 \mathrm{~mm} \mathrm{Hg}$ consistent with moderate disease. While this can sometimes be explained by a low-flow status this is often not the case. ${ }^{3}$ What then to do in that common situation?

Major interest now surrounds CT calcium scoring of the valve as an assessment of AS severity in cases where echocardiographic measurements are conflicting. ${ }^{4}$ This is of particular appeal because calcification is believed to be the predominant process driving AS progression and because it provides a flow-independent assessment of severity. ${ }^{5}$ Complex categorisation of patients based upon flow status and systolic function is therefore not required.

The last few years have witnessed rapid advances in this field. Aortic valve CT calcium scoring has demonstrated a close association with haemodynamic measures of AS on Doppler echocardiography and offers powerful prediction of future disease progression. ${ }^{2}{ }^{6}$ Moreover we now have proposed cut-offs for differentiating moderate from severe disease (men $2065 \mathrm{AU}$, women $1274 \mathrm{AU})$ that provide powerful prognostic information over and above standard echocardiographic indices. $^{7}$ However, before CT calcium scoring translates into clinical practice it is imperative that we are able to interpret its results in all the different types of patients affected by the disease. For example it has become evident that there are differences in CT calcium scoring between the sexes

Centre for Cardiovascular Science, University of Edinburgh, Edinburgh, UK

Correspondence to Dr Marc R Dweck, Chancellor's Building, University of Edinburgh, 49 Little France Crescent, Edinburgh EH16 4SB, UK; marc.dweck@ed. ac.uk, marcdweck@hotmail.com with women requiring less calcium than men to develop severe stenosis (even after indexing for body size), perhaps reflecting the hormonal effects of testosterone. ${ }^{8}$

The Heart manuscript by Shen et al ${ }^{9}$ expands on this important theme, investigating whether CT calcium scoring is likely to be of clinical use in patients with bicuspid valves and in younger patients with AS. Two hundred patients with a range of stenosis severities were prospectively recruited as a subgroup of the PROGRESSA study. No patient in the cohort had a history of rheumatic fever and all had preserved left ventricular systolic function. They underwent Doppler echocardiography and CT calcium scoring within a 3-month period, allowing comparisons to be made between the assessments of disease severity on the two scans. In particular the authors investigated the agreement between the mean transvalvular gradient on echocardiography and the aortic valve calcium density (AVCd) from CT. AVCd is a simple surrogate of the aortic valve calcium score but indexes it to the cross-sectional area of the left ventricular outflow tract to account for different valve sizes. It may offer incremental diagnostic accuracy in determining AS severity using cut-offs of AVCd $292 \mathrm{AU} / \mathrm{cm}^{2}$ in women and $476 \mathrm{AU} / \mathrm{cm}^{2}$ in men. ${ }^{2}$

Twenty per cent of the cohort had a bicuspid aortic valve and $80 \%$ a trileaflet valve. As expected the latter were older (median age 72 years vs 51 years), more likely to be male (76\% vs $56 \%$ ) and had more comorbidities. In these patients with trileaflet valves a good correlation was observed between increasing AVCd and MG $(\rho=0.61, p<0.0001)$ that persisted in both the younger ( $<72$ years) and older $(\geq 72$ years) age groups. The story however appeared different in the cohort of patients with a bicuspid aortic valve. While patients $\geq 51$ years in this group still demonstrated a strong correlation between AVCd and MG $(p=0.55$, $\mathrm{p}=0.009$ ) no correlation was observed in patients aged $<51$ years with a bicuspid aortic valve.

These observations have important implications both with respect to the utility of CT calcium scoring and the likely pathobiology underlying valve narrowing. First, it suggests that CT calcium scoring is likely to be of clinical utility in the vast majority of patients that we encounter in the clinic: subjects with trileaflet valves and patients $>51$ years with a bicuspid valve. Moreover it indicates that calcium is crucial to the pathophysiology of valve narrowing in these patients and therefore an important potential therapeutic target. ${ }^{5}$

However it also implies that CT calcium scoring may grossly underestimate the severity of AS in young patients under 50 years with a bicuspid valve. This imaging technique should therefore be approached with caution in this subgroup and probably avoided. What is the pathophysiological explanation for this anomaly? Perhaps the first question to address is whether it predominantly represents a function of the bicuspid structure of the valve or of patient age (subjects in this group were substantially younger than the patients in the trileaflet group). The authors postulate several explanations related to the former: that bicuspid valves may suffer from leaflet fibrosis rather than calcification; that abnormalities in the morphology of the leaflets and valve orifice lead to increased gradients and reduced effective orifice area; or that the eccentric jets associated with bicuspid valves cause a pressure drop in excess of the reduction in effective orifice area. While each is plausible, why these factors would affect younger patients with bicuspid valve disease but not older subjects remains unclear. The alternative explanation is that this phenomenon instead simply reflects a function of age. As discussed the patients not demonstrating a correlation between MG and AVCd were substantially younger than the other subjects in the study. The lack of calcium in the valves of these patients might therefore reflect a different pathological process in those under the age of 50 years, who also appear less prone to calcification in other conditions.

Further work in this area is required. Interestingly we recently encountered a 58-year-old female patient that might support this hypothesis. She had a trileaflet aortic valve and no history of rheumatic heart disease or hyperlipidaemia. Echocardiographic measurements were discrepant but suggested at least moderate AS (peak velocity $3.6 \mathrm{~m} / \mathrm{s}, \mathrm{MG} 27 \mathrm{~mm} \mathrm{Hg}$, AVA $0.8 \mathrm{~cm}^{2}$ ). However her CT calcium score indicated only minimal calcification (36 AU, AVCd of $12.7 \mathrm{AU} / \mathrm{cm}^{2}$ ), apparently out of keeping with the degree of her valve narrowing. A contrast CT was performed and provided explanation 




Figure 1 A 58-year-old woman with aortic stenosis investigated by echocardiography and CT. (A) Doppler echocardiography demonstrating a peak aortic valve (AV) velocity of $3.6 \mathrm{~m} / \mathrm{s}$, a mean gradient of $27 \mathrm{~mm} \mathrm{Hg}$ and an aortic valve area (AVA) of $0.8 \mathrm{~cm}^{2}$ based upon the continuity equation. (B) Non-contrast calcium scoring CT in the axial plane showing minimal aortic valve calcification (AV calcium score $37 \mathrm{AU}$ ). (C) Images from contrast-enhanced $C T$ in the en face plane and (D) coronal plane revealing marked leaflet thickening with low attenuation signal suggestive of valve fibrosis. AO, aorta; AV, aortic valve; AVA, aortic valve areas; PG, pressure gradient; VTI, velocity time integral.

demonstrating extensive valve thickening and narrowing, in the absence of calcification but with appearances consistent with fibrosis (figure 1). While anecdotal and lacking histological validation, this case illustrates that young patients may develop advanced AS in the absence of major valve calcification and supports a difference in their underlying pathobiology. Alternative imaging and therapeutic strategies may therefore be required in these patients compared with the broader population with AS.

In summary Shen et al are to be congratulated on their innovative study. Indeed there is a need for more such studies to inform about which patients with AS would benefit from CT imaging and to provide further insights into the complex pathophysiology underlying this condition.

Funding TAP, TRGC and MRD are supported by the British Heart Foundation (SS/CH/09/002/26360, FS/13/ 77/30488, SS/CH/09/002/2636, FS/14/78/31020).

MRD is the recipient of the Sir Jules Thorn Award for Biomedical Research 2015.

Competing interests None declared.

Provenance and peer review Commissioned; internally peer reviewed.

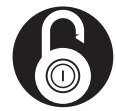

\section{OPEN ACCESS}

Open Access This is an Open Access article distributed in accordance with the Creative Commons Attribution Non Commercial (CC BY-NC 4.0) license, which permits others to distribute, remix, adapt, build upon this work non-commercially, and license their derivative works on different terms, provided the original work is properly cited and the use is non-commercial. See: http:// creativecommons.org/licenses/by-nc/4.0/

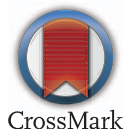

To cite Cartlidge TRG, Pawade TA, Dweck MR. Heart 2017:103:8-9.

Published Online First 19 September 2016

\section{S Linked}

- http://dx.doi.org/10.1136/heartjnl-2016-309665

Heart 2017;103:8-9.

doi:10.1136/heartjn-2016-310297

\section{REFERENCES}

1 Vahanian A, Alfieri O, Andreotti $\mathrm{F}$, et al. Guidelines on the management of valvular heart disease (version 2012). Eur Heart $J$ 2012:33:2451-96.

2 Clavel MA, Messika-Zeitoun D, Pibarot $P$, et al. The complex nature of discordant severe calcified aortic valve disease grading: new insights from combined Doppler echocardiographic and computed tomographic study. J Am Coll Cardiol 2013;62:2329-38.

3 Chin CWL, Khaw HJ, Luo E, et al. Echocardiography underestimates stroke volume and aortic valve area: implications for patients with small-area low-gradient aortic stenosis. Can J Cardiol 2014;30:1064-72

4 Chin CWL, Pawade TA, Newby DE, et al. Risk stratification in patients with aortic stenosis using novel imaging approaches. Circ Cardiovasc Imaging 2015;8:e003421

5 Pawade TA, Newby DE, Dweck MR. Calcification in aortic stenosis: the skeleton key. J Am Coll Cardiol 2015;66:561-77.

6 Nguyen V, Cimadevilla C, Estellat C, et al. Haemodynamic and anatomic progression of aortic stenosis. Heart 2015;101:943-7.

7 Clavel MA, Pibarot P, Messika-Zeitoun D, et al. Impact of aortic valve calcification, as measured by MDCT, on survival in patients with aortic stenosis: results of an international registry study. J Am Coll Cardiol 2014;64:1202-13.

8 Zhu D, Hadoke PW, Wu J, et al. Ablation of the androgen receptor from vascular smooth muscle cells demonstrates a role for testosterone in vascular calcification. Sci Rep 2016;6:24807.

9 Shen M, Tastet L, Capoulade R, et al. Effect of age and aortic valve anatomy on calcification and haemodynamic severity of aortic stenosis. Heart 2017;103:32-9. 